\title{
The Papanicolaou smear versus colposcopy for evaluation of the uterine cervix in women with perineal condyloma: A preliminary study
}

LANE K. SHIMA, D.O.
Philadelphia, Pennsylvania

Virologic, epidemiologic, and biochemical data point to the human papillomavirus (HPV) as an etiologic factor in the development of cervical cancer. Traditional screening methods for this type of carcinoma include the Papanicolaou (Pap) smear as the initial testing procedure. In this study of 8 women with perineal condyloma, 7 had negative Pap smears. However, on colposcopic examination and biopsy of these patients, 6 were demonstrated to have histologic evidence of HPV infection of the cervix, and an additional patient exhibited dysplastic changes. Therefore, in 7 of the 8 women, the Pap smear was not a sensitive enough screening tool to detect these abnormalities. The author advocates the use of colposcopy with directed biopsy as the primary screening modality for women with perineal condyloma.

The incidence of cervical cancer has undergone a dramatic decline since the advent of widespread screening of women utilizing the Papanicolaou (Pap) smear of the uterine cervix. The test has identified women for whom further evaluation, that is, colposcopy with directed biopsy, is warranted.

It is well documented that the Pap smear is an excellent screening tool when a positive result is reported. Unfortunately, however, overreading of the cytologic smear may occur. In a retrospective study of colposcopically directed cervical biopsies, Binder and associates ${ }^{1}$ found a 57.1 percent incidence of grade 3 cervical intraepithelial neoplasia diagnosis when, actually, only condyloma was present. Of those overdiagnosed women, 53.6 percent underwent cone biopsy or hysterectomy when a less radical procedure might have been sufficient. At the other end of the spectrum, the false negative rate has been reported to be as high as $20-40$ percent. $^{2}$

The present prospective study has been undertaken to evaluate the latter situation. Is the Pap smear a sensitive enough tool to detect early human papillomavirus (HPV) lesions of the cervix (possible etiologic agents for squamous cell carcinoma) in women who have grossly visible evidence of an HPV infection of the external genitalia?

\section{Method}

Between September 1 and December 1, 1985, the Department of Obstetrics and Gynecology of the Philadelphia College of Osteopathic Medicine asked our referral base of physicians to refer women with condyloma acuminata lesions of the perineum and perianal areas to our colposcopy clinic. Eight women who fulfilled the study criteria during this time period comprised the study group. For each patient, evaluation of the cervix consisted of two procedures: First, an endocervical cotton applicator was utilized in conjunction with an Ayre spatula to obtain the Pap smear material. Each slide was immediately fixed and sent for cytologic evaluation. Second, colposcopy of the uterine cervix was performed with a Zeiss colposcope following 
the application of 3 percent acetic acid to the cervix, and biopsy specimens of exophytic and acetowhite lesions of these areas were obtained. Endocervical curettage was performed in conjunction with all colposcopies. The specimens were fixed in formalin and sent for histologic evaluation. Then the cytologic and histologic findings were compared.

\section{Results}

For the purpose of this study, a Pap smear was considered to be negative when there was no evidence of dyskeratosis, koilocytosis, or dysplasia. Of the 8 women in the study, 7 had negative Pap smears, while the remaining patient had a class IIR smear (atypical squamous metaplasia or hyperkeratosis). All of the women had either exophytic lesions or areas that appeared acetowhite on colposcopy. Biopsy revealed 5 women to have histologic evidence of koilocytosis, 1 of acanthosis, 1 of chronic inflammation, and 1 of moderate dysplasia.

\section{Discussion}

The rapidly decreasing incidence of cervical cancer and the concomitant increase in the detection of less severe lesions have been attributed to widespread routine screening of the uterine cervix. Early detection has allowed clinicians to use more conservative modes of therapy to intervene at a much earlier stage in the disease process, which has resulted in improved survival rates. With selective evaluation of patients at high risk for dysplastic changes, it is thought that the mortality rate will drop further.

Review of the literature has revealed colposcopy to be an effective adjunctive tool in the evaluation of the atypical Pap smear. The preliminary data obtained in the present study support the thesis that all women with visible evidence of perineal condyloma should directly undergo colposcopy with directed biopsy for evaluation, regardless of Pap smear results. Although the study population was not large enough for statistical significance, the finding that 5 of the women had koilocytotic atypia, and 1 patient had moderate dysplasia, even though all 6 had negative Pap smears, does seem to demonstrate a trend. In these 6 women, no follow-up treatment would have been offered if only Pap smear results had been considered. With transition times from normal Pap smears to CIN I-III appearing to be shorter than previously believed (Table l), ${ }^{3}$ a lack of appropriate treatment for these 6 women, coupled with the possibility of poor patient compliance with the recommendation of annual Pap smears, may have severe consequences with regard to morbidity and mortality.

Definitive elucidation of the cause(s) of cervical cancer has yet to be accomplished. Epidemiologic,
TABLE 1. DURATION OF TRANSITION FROM NORMAL PAP SMEAR TO CIN-I-III DISEASE (MODIFIED FROM NOUMOFF ${ }^{2}$ ).

\begin{tabular}{|lc|}
\hline Transition & $\begin{array}{c}\text { No. of years } \\
\text { (mean) }\end{array}$ \\
\hline Normal to mild-moderate & 1.62 \\
Normal to moderate-severe & 2.20 \\
Normal to carcinoma in situ & 4.51
\end{tabular}

virologic, and clinicopathologic evidence strongly support a venereal mode of transmission. Rotkin ${ }^{4}$ demonstrated that low socioeconomic status, early onset of sexual activity, and multiple sexual partners all have positive correlations with the incidence of cervical cancer.

Meisels and Morin ${ }^{5}$ found a high incidence of condylomatous changes in the cytologic specimens they studied; in a randomized screening of 234,715 women, 1.69 percent of Pap smears demonstrated this abnormality. This was consistent with data from Reid and associates, ${ }^{6}$ who reported that 92 of 7,281 routine smears ( 1.26 percent) demonstrated evidence of HPV infection. Meisels and Morin ${ }^{5}$ also noted a coexistence of dysplastic and neoplastic changes, with condylomata present 25.6 percent of the time.

Of the 41 distinct types of HPV identified to date, types $6,11,16,18,31$, and 33 have been determined to cause genital tract infections. ${ }^{7,8}$ Of these, types 6 and 11 are commonly found in acuminate warts, but they have seldom been identified in invasive squamous cell cancer, while types $16,18,31$, and 33 have been implicated in malignant transformation of the uterine cervix. ${ }^{8-10}$ Genetic sequences homologous to the genome of types $16,18,31$, and 33 have been obtained from cells from women with cervical intraepithelial neoplasia or invasive carcinoma. ${ }^{7-10}$

Of importance to the light microscopist is the close association between flat warts with abnormal mitotic figures and HPV 16, which have a greater risk of progression to higher grade lesions than diploid or polyploid lesions without abnormal mitotic figures. ${ }^{8}$ Therefore, merely on the basis of light microscopic findings, a pathologist can derive some predictive value from the biologic feature of aneuploidy and the morphologic characteristic of abnormal mitotic figures in determining the possibilities for neoplastic change. ${ }^{7}$

\section{Summary}

This paper proposes that while the Papanicolaou smear of the uterine cervix is an excellent screening tool for the general female population, it may have a notable deficiency for the subset of women who have perineal condyloma. In this preliminary 
study, the Pap smear failed to demonstrate histologically proved abnormalities in 6 of 8 cases. Thus, it is recommended that all women with visible condylomatous lesions have colposcopy with directed biopsies as their primary screening method. These lesions could then be treated early and effectively with conservative measures.

This study is ongoing, and further reports are forthcoming.

Appreciation is expressed to Patrick Becher, D.O., for his assistance in this study.

1. Binder, M.A., et al.: The changing concepts of condyloma. A retrospective study of colposcopically directed cervical biopsies. Am J Obstet Gynecol 151:213-9, 15 Jan 85

2. Coppleson, L.W., and Brown, B.: Estimation of the screening error rate from the observed detection rates in repeated cervical cytology. Am J Obstet Gynecol 119:953-8, 1 Aug 74

3. Noumoff, J.: Lecture. Philadelphia Obstetrical Society Meeting, Philadelphia, December 12, 1985
4. Rotkin, I.D.: A comparison review of key epidemiological studies in cervical cancer related to current searches for transmissible agents. Cancer Res 33:1353-67, 1973

5. Meisels, A., and Morin, C.: Human papillomavirus and cancer of the uterine cervix. Gynecol Oncol 12:S111-23, Oct 81

6. Reid, R., et al.: Noncondylomatous cervical wart virus infection. Obstet Gynecol 55:476-83, Apr 80

7. Paavonen, J.: Colposcopic findings associated with human papillomavirus infection of the vagina and the cervix. Obstet Gynecol Surv 40:185-9, Apr 85

8. Crum, C.P., et al.: Human papillomavirus type 16 and early cervical neoplasia. N Engl J Med 310:880-3, 5 Apr 84

9. Lorincz, A.T., Lancaster, W.D., and Temple, G.F.: Cloning and characterization of the DNA of a new human papillomavirus from a woman with dysplasia of the uterine cervix. J Virol 58:225-9, Apr 86

10. Beaudenon, S., et al.: Novel type of human papillomavirus associated with genital neoplasias. Nature 321:246-9, 15-21 May 86

Dr. Shima is a resident in obstetrics and gynecology at the Philadelphia College of Osteopathic Medicine, Philadelphia. Michael Mansi, D.O., FACOOG, is chairman and professor, Department of Obstetrics and Gynecology at PCOM.

Dr. Shima, PCOM, 4150 City Avenue, Philadelphia, Pennsylvania 19131 


\section{In every small town, you'll find a church, an American flag, and someone wholl sell you cocaine.}

It's a sad fact, but a fact nonetheless. Substance abuse is so widespread these days that buying cocaine is almost as easy as buying a hot dog or a new pair of shoes.

As a physician, you're in the unique position to detect drug abuse in your patients, using your training, thorough work-ups, and advanced diagnostic techniques.

Through increased detection, you can help curb this growing problem, and help make our towns (both small and large) a home for people, not pushers.

Looking for drug abuse-make it a habit.

Partnership for a Drug-Free America

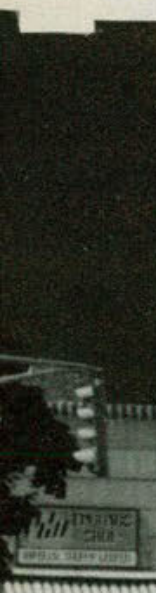

1

1

1)

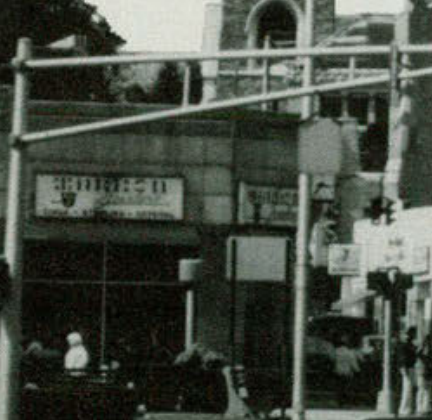

(1)

.

Hiv.
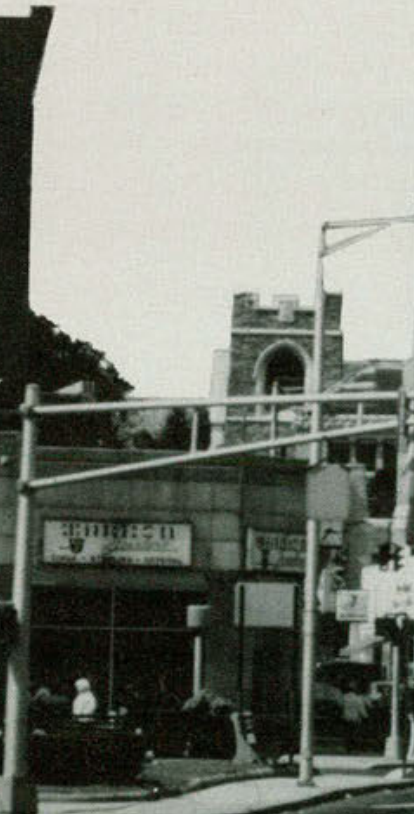